\title{
Deformation of limit cycle under perturbations
}

\author{
K. L. Chen ${ }^{a}$, Z. Z. Sun ${ }^{a}$, S. Yin ${ }^{a}$, Y. Q. Wang ${ }^{b}$, X. R. Wang ${ }^{a, *}$ \\ ${ }^{a}$ Physics Department, The Hong Kong University of Science and Technology, \\ Clear Water Bay, Hong Kong SAR, People's Republic of China \\ ${ }^{\mathrm{b}}$ Key Laboratory of Material Physics, Institute of Solid State Physics, Chinese \\ Academy of Sciences, Hefei, People's Republic of China
}

\begin{abstract}
The robustness of limit cycles of nonlinear dynamical systems is investigated by adding a small random velocity field to the famous van der Pol (VDP) equation in its two-dimensional phase plane. Our numerical calculations show that a limit cycle does not change much under a weak random perturbation. Thus it confirms the conjecture that a limit cycle will make only a small deformation under an external perturbation. The idea can be used to understand ac response of self-sustained oscillations in nonlinear dynamical systems.
\end{abstract}

Key words: superlattice, vertical transport, nonlinear, limit cycle

Following the discovery of the self-sustained current oscillations (SSCOs) $[1,2]$ in superlattices (SLs), a large number of experimental and theoretical studies have focused on different aspects of the SSCOs [3-5]. Recently, it was shown [6] that SSCOs can be understood as the manifestation of one-dimensional attractor - limit cycle, which is a fundamental concept of nonlinear science. A limit cycle is a special trajectory of an isolated closed curve of a system in its phase space in which each point corresponds to a unique state. The velocity of the system on the curve is along its tangent direction. Thus, the system will move on the curve indefinitely, giving rise to a self-sustained periodic motion. On the contrary, a point with zero velocity in the phase space is called a fixed point. The limit cycle concept not only provides a simple physical picture on SSCOs in SLs, but also gives a quantitative understanding of the frequencylocking in the ac-response of SSCO [7]. In a way, the limit cycle understanding of ac-response of SSCO is a geometrical interpretation of Arnold analysis[8].

\footnotetext{
* Corresponding author. Tel: +852-23587488; Fax: +852-23581652.
}

Email address: phxwan@ust.hk (X. R. Wang).

Preprint submitted to Elsevier Science $\quad 6$ May 2005 
This geometrical interpretation is based on a conjecture: A limit cycle is a basic object of a nonlinear dynamical system, and an external ac-field exerts its effect on the SSCO through the modification of the limit cycle. Under a weak external force, a limit cycle can deform with or without a topological change [7]. Though the conjecture is appealing, it has not, nevertheless, been tested on a model system.

In this paper, we would like to use the famous van der Pol (VDP) equation to test the conjecture. We shall add a small random velocity field to that of a pure VDP equation. Then we shall numerically follow the trajectory of the system with different starting points in the phase space. All trajectories will eventually settle down to the same isolated closed curve no matter where they start. Furthermore, this closed curve is not too far from the original limit cycle of the pure VDP equation.

The VDP equation is mathematically defined as

$$
\left\{\begin{array}{l}
\dot{x}=y \\
\dot{y}=-a\left(x^{2}-1\right) y-x
\end{array}\right.
$$

where $x, y$ are two dynamical variables and $a$ is a control parameter describing dissipative term. This is a two-dimensional autonomous nonlinear dynamical system, which can describe self-sustained oscillations in many natural dissipative systems, including those in nonlinear $L C R$ electric circuits and chemical reactions. It is well known that the equation has a typical limit cycle oscillation for a positive $a[9,10]$. Figure 1 demonstrates the limit cycle solution of VDP equation for $a=0.1$. Two trajectories starting from $(1,0)$ and $(3,0)$, respectively, end up in the same isolated closed curve - limit-cycle, around a circle of radius 2. As $a$ increases, the shape of the limit cycle changes from a circle to a square-like loop, and the corresponding self-sustained oscillation becomes pulse-like from a sinusoidal one [11].

In order to show that the limit cycle is structurally stable against a perturbation, we add a random velocity field to the right hand side of VDP system (1). The new dynamical system becomes

$$
\left\{\begin{array}{l}
\dot{x}=y+b \cdot r_{1}(x, y) \\
\dot{y}=-a\left(x^{2}-1\right) y-x+b \cdot r_{2}(x, y)
\end{array}\right.
$$

where $r_{1}(x, y)$ and $r_{2}(x, y)$ are two random functions, depending only on position $(x, y)$ in phase plane, and $b$ controls the strength of the perturbation. Obviously, system (2) cannot be solved analytically. However, one can easily solve it numerically for a given perturbing velocity field $r_{1}(x, y)$ and $r_{2}(x, y)$. To do so, we replace the continue phase space with a discrete lattice by dividing the phase plane confined inside $|x|<3$ and $|y|<3$ into $600 \times 600$ meshes. 
At each mesh site, two random numbers uniformly distributed in $[-1,1]$ are generated for $r_{1}(x, y)$ and $r_{2}(x, y)$. We shall employ the fourth-order RungeKutta method in our numerical calculations. To show the existence of and the robustness of the limit cycle solution of system (2), we plot numerically the trajectories of various perturbation strengths with different initial conditions.

Figure 2 demonstrates the trajectories of the new system. Two trajectories starting from $(1,0)$ and $(3,0)$, respectively, end up in the same isolated closed curve. A comparison of figure 2 and figure 1 shows that this closed curve is not too far from the original limit cycle of the pure VDP equation. It is because that a small random function can only change slightly the direction of velocity field in phase space. The value of velocity on limit cycle is about $v=\sqrt{\dot{x}^{2}+\dot{y}^{2}} \approx 2$ when $b<<v$ and $a$ is small. The system will be mainly guided by original vector field. Thus the numerical calculation verifies that a limit cycle does not change much when the random perturbation is small enough compared with the original velocity field.

Figure 2 also shows that a trajectory starting from $(0.1,0)$ ends up in a closed curve, localized near the origin. This closed loop around the origin is an artificial result of our model. This is because the original velocity field is perturbed in the same way everywhere in the phase plane. Since the magnitude of the velocity around the origin is proportional to its distance $r$ from the origin, thus the velocity in the region of $r<|b|$ is greatly perturbed by the random field. The phase velocity in this region is almost random. In another word, the larger $b$ is, the more sites can be regarded as having a random velocity. When $b$ is fixed, more sites have random velocity if one divides the plane into more meshes. As a result, there is a probability that a trajectory will be localized around the origin. In $2 \mathrm{D}$, all trajectories would be localized if the velocities at all sites are completely random. This is the origin of the closed curve around the origin in Fig. 2. This is demonstrated by the disappearance of the closed loop as $b$ is reduced below 0.06 as shown in Fig. 3. Then the trajectory starting from $(0.1,0)$ end up eventually in the limit cycle of the system.

The explanation of the localized loop around the origin is further confirmed by the numerical results for $b=0.1$ on a larger mesh size lattice. Instead of dividing the area of $|x|<3$ and $|y|<3$ into $600 \times 600$ meshes, we divide it into $6 \times 6$ meshes. As explained early, the probability to obtain a localized loop around the origin should be dramatically reduced. Indeed, this is what showed in Figure 4 for a typical configuration. There is no localized loop near the origin in the figure. Instead, the trajectory starting from $(0.1,0)$, ends up in the limit cycle of the system.

So far, we have considered the random perturbation on the limit cycle located in the phase plane where the phase velocity is much larger than the 
perturbation, or a case that the system is far from the bifurcation point. Near a bifurcation point, a random perturbation can greatly change the velocity field around a limit cycle. The response of such a system to an external perturbation will be much more complicated as it was demonstrated in the granular flow system[12].

It is important to notice that a limit cycle is a structurally stable object. This is because a dynamical system with a limit cycle solution must be nonlinear and non-conservative (dissipative). For a given dynamical system, the phase space can be divided into energy gain and loss regions. The system can either dissipate or gain the energy when it is away from the limit cycle, which is a consequence (compromise) of the competition between the energy gain and loss. Adding a small perturbation to the dynamical equations, the boundary between the energy gain and loss regions will move slightly. As a result, a limit cycle tries to sustain its original shape under a weak perturbation, and it just makes a small deformation. As conjectured in Ref. [7], a small deformation can occur with and without topological change in which the winding number can vary. In this study, we have only seen the deformation without topological change. This is because that a VDP equation is a two-dimensional dynamical system, and it is possible to wind a closed curve twice without a crossing in two-dimensional phase plane. One needs to use a higher dimensional model, such as those for superlattices, to observe a deformation with topological change.

The verification of the conjecture about limit-cycle responses to an external perturbation is important in understanding the ac-response of nonlinear dynamical system. As what we showed early in Ref. [7], one can understand the frequency-locking in the SSCO based on the conjecture. The same idea may also be applicable to the networks in molecular biology, where a central problem is to understand gene regulatory networks and how they collectively operate with remarkable precision in response to environmental cues [13]. In principle, the way of thinking can be applied to other problem, such as response to an external quasi-periodic or random force, and how a chaotic or turbulent system responds to an external force. In the case of aperiodic effect, there are two possible ways of exerting a quasi-periodic force. One is to replace the periodic SL by a quasi-periodic or random SL. This quasi-periodicity effect had been extensively studied, and many interesting results regarding the electronic structure were obtained[14-16]. The other less studied quasi-periodicity effect is how a system responds to a quasi-periodic or random external force. This is a problem that we would like to study in the future. In the case of response of a chaotic or turbulent system to an external force, one may attack this problem in the same spirit as what we did in understanding frequencylocking in SSCO. Of course, it requires a precise understanding and description of chaos and turbulence that is lacking at the moment[17]. 
In summary, the response of the limit cycle in the van der Pol (VDP) equation under a weak random perturbation on the velocity field in phase plane is studied. Our result shows that limit cycle does not change much when the random perturbation is small compared with the original velocity field. This verifies our early conjecture: A limit cycle is not easy to be destroyed, and it will try to sustain its original shape under a weak perturbation.

\section{Acknowledgements}

This work is supported by UGC, Hong Kong, No. 603403.

\section{References}

[1] S. H. Kwok, T. B. Norris, L. L. Bonilla, J. Galán, J. A. Cuesta, F. C. Martínez, J. M. Molera, H. T. Grahn, K. Ploog, R. Merlin, Phys. Rev. B 51 (1995) 10171; H. T. Grahn, J. Kastrup, K. Ploog, L. L. Bonilla, J. Galán, M. Kindelan, and M. Moscoso, Jpn. J. Appl. Phys. 34 (1995) 4526; J. Kastrup, R. Klann, H. T. Grahn, K. Ploog, L. L. Bonilla, J. Galán, M. Kindelan, M. Moscoso, R. Merlin, Phys. Rev. B 52 (1995) 13761.

[2] J. N. Wang, B. Q. Sun, X. R. Wang, and H. L. Wang, Solid State Commun. 112 (1999) 371; Jiannong Wang, Baoquan Sun, Xiangrong Wang, Yuqi Wang, Weikun Ge, and Hailong Wang, Appl. Phys. Lett. 75 (1999) 2620; X. R. Wang, J. N. Wang, B. Q. Sun, and D. S. Jiang, Phys. Rev. B 61 (2000) 7261.

[3] J. Kastrup, R. Hey, K. H. Ploog, H. T. Grahn, L. L. Bonilla, M. Kindelan, M. Moscoso, A. Wacker, J. Galán, Phys. Rev. B 55 (1997) 2476; A. Wacker, M. Moscoso, M. Kindelan, and L. L. Bonilla, Phys. Rev. B 55 (1997) 2466; E. Schomburg, K. Hofbeck, R. Scheuerer, M. Haeussler, K. F. Renk, A.-K. Jappsen, A. Amann, A. Wacker, E. Schöll, D. G. Pavel'ev, and Yu. Koschurinov, Phys. Rev. B 65 (2002) 155320.

[4] A. Wacker, Phys. Rep. 357 (2002) 1; L. L. Bonilla, J. Phys.: Condens. Matter 14 (2002) R341.

[5] Yaohui Zhang, R. Klann, K. H. Ploog, and H. T. Grahn, Appl. Phys. Lett. 70 (1997) 2825; K. J Luo, S. W. Teitsworth, H. Kostial, H. T. Grahn, and N. Ohtani, Appl. Phys. Lett. 74 (1999) 3845.

[6] X. R. Wang, and Q. Niu, Phys. Rev. B. 59 (1999) R12755; X. R. Wang, Z. Z. Sun, S. Q. Duan, and S. D. Wang, cond-mat/0211306.

[7] Z. Z. Sun, H. T. He, J. N. Wang, S. D. Wang, and X. R. Wang, Phys. Rev. B 69 (2004) 045315. 
[8] V. I. Arnold (ed.), Dynamical Systems, 2nd ed., Springer-Verlag, 1993; V. I. Arnold, Mathematical Methods of Classical Mechanics, 2nd ed., SpringerVerlag, 1989.

[9] R. Seydel, Practical Bifurcation and Stability Analysis, 2nd ed., SpringerVerlag, 1994.

[10] S. H. Strogatz, Nonlinear Dynamics and Chaos: with applications to physics, biology, chemistry, and engineering, Addison-Wesley, 1994.

[11] R. H. Rand, Lecture Notes on Nonlinear Vibrations, Version 40, http://www.tam.cornell.edu/randdocs, Cornell University.

[12] V. G. Benza, F. Nori and O. Pla, Phys. Rev. E 48 (1993) 4095.

[13] R. Albert and A. L. Barabai, Rev. Mod. Phys. 74 (2002) 47.

[14] S. Tamura and F. Nori, Phys. Rev. B 40 (1989) 9790; S. Tamura and F. Nori, Phys. Rev. B 41 (1990) 7941.

[15] M. Kolář and F. Nori, Phys. Rev. B 42 (1990) 1062; M. Koláŕ, M. K. Ali and F. Nori, Phys. Rev. B 43 (1991) 1034.

[16] Q. Niu and F. Nori, Phys. Rev. B 42 (1990) 10329.

[17] By precise understanding, we mean that we know properties of each point in a strange attractor, not just its dimensionality. For example, we know that the phase velocity at each point on a limit cycle is along its tangent direction, and the velocity at a fixed point is zero. However, we have no much knowledge about the phase velocity on a strange attractor.

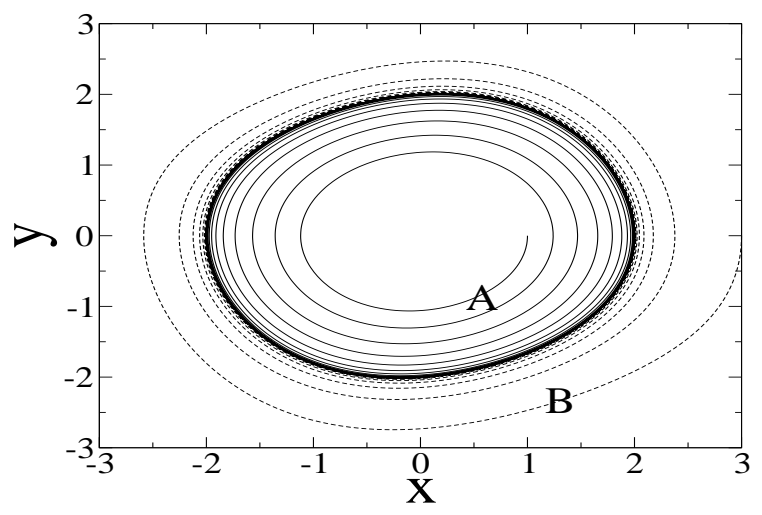

Fig. 1. Trajectories of the VDP equation for $a=0.1$. Curve $A$ is a trajectory that starts from $(1,0)$; curve $B$ is a trajectory that starts from $(3,0)$. Two trajectories end up in the same isolated closed curve - limit cycle, around a circle of radius 2. 


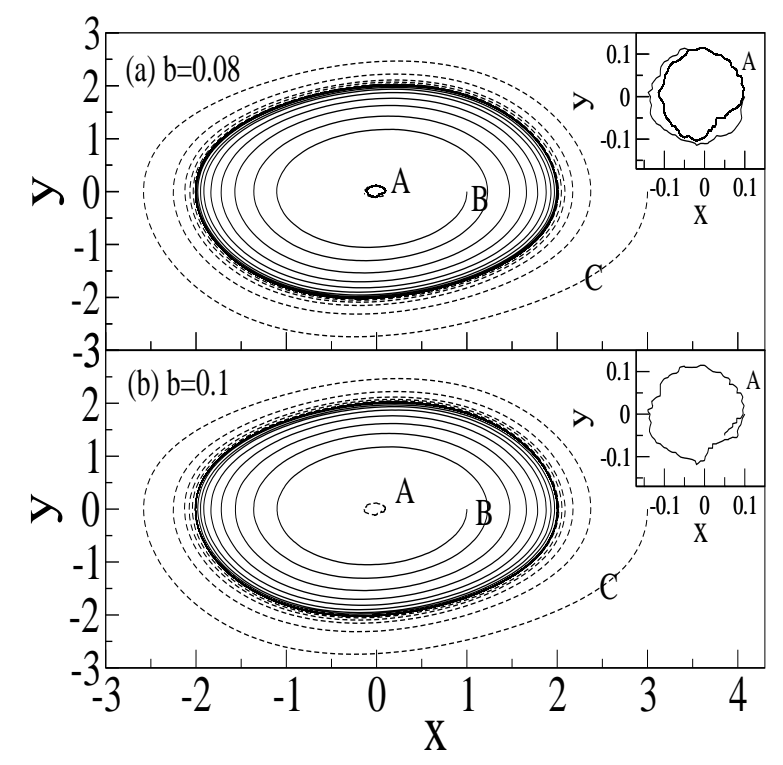

Fig. 2. Trajectories of the VDP equation for $a=0.1$ under a random perturbation. The continue phase plane of $|x|<3$ and $|y|<3$ is divided into $600 \times 600$ meshes. (a) $b=0.08$, curve $A$ is a trajectory starting from $(0.1,0)$, localized near the origin; curve $B$ is a trajectory starts from $(1,0)$; curve $C$ is a trajectory starts from $(3,0)$. Curve $B$ and curve $C$ end up in a closed curve, around a circle of radius 2; (b) $b=0.1$, it has Similar result as (a).

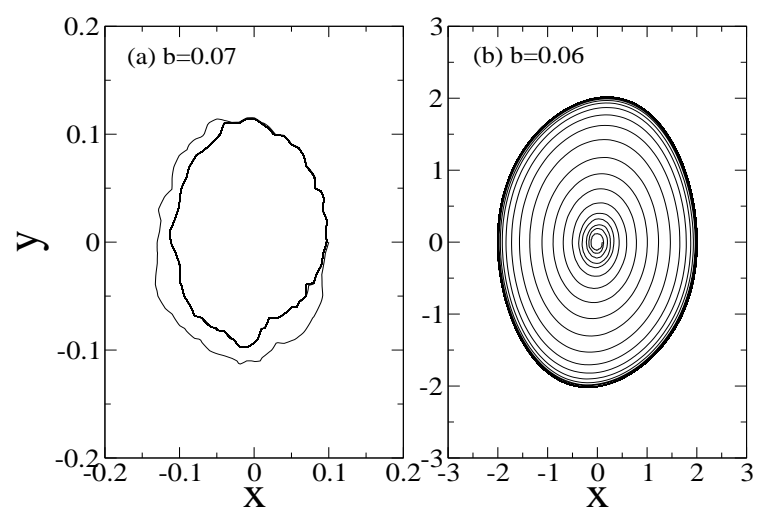

Fig. 3. The trajectories start from $(0.1,0)$. The continue phase plane of $|x|<3$ and $|y|<3$ is divided into $600 \times 600$ meshes. (a) The trajectory is localized around the origin for $b=0.07$; (b) the localized loop around the origin disappears for $b=0.06$. The trajectory settles down eventually to its limit cycle. 


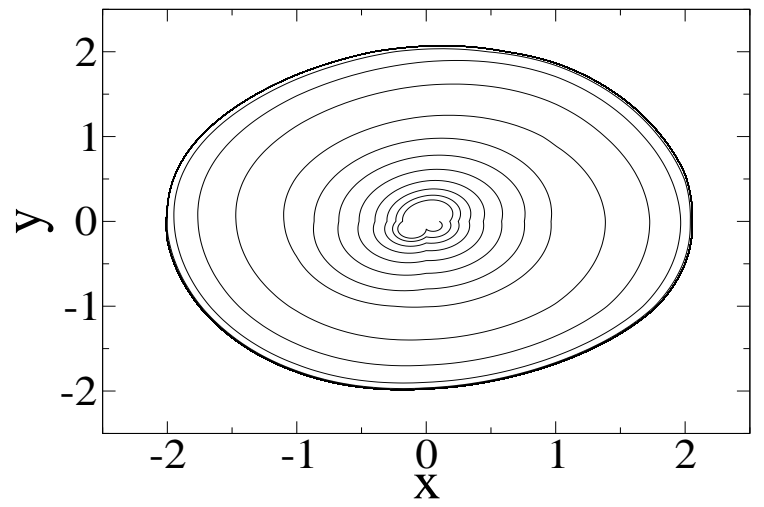

Fig. 4. The trajectory of the VDP equation for $a=0.1$ under a random perturbation with $b=0.1$. It starts from $(0.1,0)$. The continue phase plane of $|x|<3$ and $|y|<3$ is divided into $6 \times 6$ meshes. 